\title{
Psychotherapie als kulturelles Phänomen
}

\author{
Eine Psychotherapie entsteht auf dem Boden einer spezifischen gesellschaftlichen \\ Situation, wird geprägt von deren Menschenbild und durch einen Anstoss, der die \\ Neuschöpfung einer Form von Psychotherapie hervorruft. Diese Regel gilt für alle \\ Formen von Psychotherapien, die im 20. Jahrhundert entstanden sind.
}

Die Psychotherapie schafft in der Regel eine therapeutisch wirksame Beziehung zwischen Therapeut und Patient, die nicht abgelöst werden kann von deren jeweiligem gesellschaftlichem Hintergrund.

Wir stimmen überein mit der Feststellung von A. W. Schuch, der erklärt: «Die Tatsache der Ausprägung der konkreten Formen und Verfahren der Psychotherapie ist zuerst spezifischer Ausdruck der Veränderung bestimmter historisch-gesellschaftlicher Bedingungen. Die Entstehung und Entwicklung von Psychotherapie ist im Zusammenhang mit der historisch zunehmenden Exposition des Individuums zu sehen. Diese veränderten historisch-gesellschaftlichen Bedingungen prägen in hohem Masse die Formen und Verfahren von Psychotherapie, wie auch das psychotherapeutische Denken.»

Für das Verständnis der Psychiatriegeschichte des 20. Jahrhunderts und der Entstehung einer grossen Zahl verschiedener Formen von Psychotherapien ist es nötig, einen Blick auf die Geschichte des 20. Jahrhunderts zu werfen.

Das 20. Jahrhundert in der westlichen Welt ist durch grosse Gegensätze gekennzeichnet. Es ist ein Zeitalter grossen kulturellen Fortschritts mit der Entdeckung bedeutender technischer Neuerungen auf den verschiedensten Gebieten. Das 20. Jahrhundert hat es möglich gemacht, Menschen auf den Mond zu schicken, hat die Transplantationschirurgie zu einem Höchststand gebracht, und eine grosse Zahl psychischer und somatischer Krankheiten wurden durch neue Medikamenten heilbar, und nicht zuletzt hat man es verstanden, das menschliche Genom zu entschlüsseln. Die Entdeckungen auf dem Gebiete der Telekommunikation haben eine neue Epoche im Wirtschaftsbereich und der Sozialbeziehungen herbeigeführt.

Das Zeitalter des beinahe grenzenlosen technischen Fortschritts war aber auch ein Zeitalter des Verlustes aller humanen Werte in der Folge von Kriegen mit Millionen von Opfern. Die Wirtschaftswelt erfuhr neben einem starken Aufschwung vorübergehende Zusammenbrüche. In dieser Welt der Gegensätze und der Zerrissenheit hat auch der einzelne Mensch eine Veränderung durchgemacht. Das Individuum des Endes des 20. Jahrhunderts hat sich in vielem verändert gegenüber dem Menschen am Anfang des Jahr- hunderts. In mehrfacher Hinsicht haben die Menschen an Freiheit gewonnen, aber gleichzeitig an Autonomie verloren.

Die im 20. Jahrhundert entwickelten Psychotherapien widerspiegeln die Umwelt, in der sich deren Entdecker befunden haben. S. Freud behandelte Patienten, die durch die patriarchalische familiäre Struktur des viktorianischen Zeitalters in ihrem Individualisierungsprozess behindert worden waren und an sexuellen Störungen litten, die sich auf vielfachen somatischen Wegen äusserten, häufig durch Hysterie mit ihren dramatischen Konversionssymptomen. Störungen in der frühkindlichen Entwicklung, wie sie Freud häufig zu Gesicht bekam, wie hysterische Lähmungen oder Anfälle, führten zu seinen entscheidenden Entdeckungen. Aus seinen Erfahrungen mit den Patienten und deren unterdrücktem Sexualleben entwickelte er die Theorie von der Sexualenergie, die das Seelenleben dominiere, vor allem durch deren Präsenz im Unbewussten.

C.G. Jung kritisierte die Auffassungen Freuds als einseitig, was ihn veranlasste, seine eigene Libidotheorie zu entwickeln und ein Menschenbild zu entwerfen, das mit ursprünglichen geistigen Aspirationen in Verbindung gebracht wurde. Freud und Jung verkörpern durch ihre Theorien den Gegensatz, der sich durch das ganze 20. Jahrhundert hinwegzieht: den kollektiven Konflikt zwischen Barbarei und elementarer Triebhaftigkeit gegenüber dem Streben nach Höherentwicklung in Wissenschaft und Kultur.

Das Aufkommen eines inhumanen Totalitarismus in Deutschland beeinflusste wahrscheinlich Alfred Adler in seinen Aussagen, die als eine Art von Beschwörungen interpretiert werden können, den Menschen als solchen mit seiner Zwiespältigkeit d.h. mit seinen Schwächen und gleichzeitig seinem Willen zum Guten zu erkennen. Adler führte eine Zukunftsperspektive in seine Analysen ein, indem er versuchte, den Menschen in seiner Bewegung auf ein Ziel hin zu orientieren. Dieses Ziel blieb jedoch streng an die Persönlichkeit des Patienten gebunden, indem der «neurotische Charakter» als «Diener eines fiktiven Zweckes» beschrieben wurde.

Die Dilemmata zwischen Individuum oder Gesellschaft und zwischen einer biologischen oder soziologischen theoretischen Konzeption hat die systemische 
Therapie zu bewältigen versucht durch eine Vereinigung der unterschiedlichen Ansätze. Auf der Metatheorie von L. von Bertalanffi beruht folgende Aussage: «Ein System ist hierbei eine Einheit aus Elementen - mehr als deren blosse Summe. Die Elemente haben untereinander und zu anderen Systemen Beziehungen: Solche Wechselwirkungen können selbstverständlich über die Eigenschaften der Elemente Hinausgehendes bewirken.» Obwohl im Ansatz positiv und konstruktiv, sind der Systemischen Therapie enge Grenzen gesteckt, indem ihr Interaktionsmodell kaum über den familiären Rahmen hinausreicht.

Die Menschen werden seit jeher nicht von irgendwelchen philosophischen Denkern in ihrer Lebensgestaltung bestimmt, sondern von den Realitäten, die sie im Rahmen ihrer Lebensbewältigung antreffen. In diesem Sinne sind die geistig-kulturellen Strömungen des Modernismus und des Postmodernismus keine von Philosophen entwickelten Konzepte, sondern die Beschreibung prozesshafter Tatbestände der Menschheitsgeschichte.

Erich Fromm hat sich sicher niemals die Frage gestellt, ob er der Epoche der Moderne oder Postmoderne zuzurechnen wäre. Geprägt von seiner geistigen und sozialen Herkunft und unter dem Eindruck der Welt, in der er lebte, hat er eine Stellung bezogen, die sein psychotherapeutisches Werk gestaltet hat. Seine humanistische und soziale Art, die Gesellschaftsprobleme zu betrachten, ist nicht einer Willensentscheidung zu verdanken, sondern einer geistig-moralischen Reaktion auf die gesellschaftliche Situation seiner Epoche, die ihn auch dazu veranlasste, der Geistesrichtung des Marxismus zu folgen. Unbewusst hat Fromm durch seine Zugehörigkeit zum Marxismus auch die Greuel des Nationalsozialismus zu bewältigen versucht. Die Aussagen Fromms haben so gesehen verschiedene Wurzeln, einerseits in einem unbewussten Abwehrprozess gegen eine nicht akzeptable inhumane Zivilisation und andererseits in einem intellektuellen Erkenntnisprozess.

Eine Psychotherapie entsteht auf dem Boden einer spezifischen gesellschaftlichen Situation mit deren Menschenbild und durch einen evolutiven Anstoss, der die Neuschöpfung einer bestimmten Form von Psychotherapie hervorruft. Diese Regel gilt für alle Formen von Psychotherapien des 20. Jahrhunderts. Sie hätten sich nicht aus dem Nichts entwickeln können, sondern sind immer dank mehrerer Voraussetzungen entstanden. Von dieser Regel machte auch der Behaviorismus keine Ausnahme, der die psychischen Funktionen als zentralnervöse Vorgänge zu erfassen und erklären versucht. Die Entwicklung der Verhaltenstherapie wurde ermöglicht durch die Fortschritte im Verständnis der neurophysiologischen Funktionen und durch das spezifische Menschenbild dieser Therapierichtung, die für die Behandlung des Menschen keiner seelischen Funktionen bedurfte.

Die Anfang des 2. Weltkrieges ausgewanderten Psychiater kehrten manchmal nach Deutschland zurück, wo sie hofften, sich eine neue Existenz auf- zubauen. Wie könnte man sich vorstellen, dass die durchgemachten schrecklichen Erlebnisse diese Menschen, die den Krieg überlebt hatten, in ihrem Kern unbeeinflusst liessen? Die Rückwanderer mussten einen Weg finden, um die Vergangenheit zu verarbeiten. Schaut man die Werke psychotherapeutischer Autoren an, findet man häufig einen Aufruf zum Glauben an den Sinn und das Gute. Besonders eindrücklich ist in dieser Hinsicht das Werk von Viktor Frankl, der mit grossem intellektuellem Talent die horrenden Erfahrungen zu verarbeiten versucht durch den Aufruf, nach dem Sinn im Leben zu suchen. Er bezeichnet transzendentale geistige Bedürfnisse des Menschen als von zentraler Bedeutung. Die Auswirkungen eines solchen Menschenbildes sind zweifellos konstruktiv gewesen, ebenso wie die Auffassungen vom Menschen als eines Gemeinschaftswesens, wie sie uns durch Günther Ammon vermittelt worden sind. Auch im Werk von Ammon erkennt man das Bedürfnis, an positive Entwicklungsmöglichkeiten und an das Gute im Menschen zu glauben, der durch seine Interaktion mit der Gesellschaft die Energie erhalten sollte, sich zu vervollkommnen.

Wir haben nun die Grenzen des 20. Jahrhunderts zum 21. Jahrhundert überschritten und es stellt sich die Frage, inwiefern die Menschenauffassungen, die im vergangenen Jahrhundert Eingang in die therapeutischen Methoden gefunden haben, heute nicht einer Anpassung an eine neue gesellschaftliche Situation und eine neue Stellung des Individuums in derselben bedürften. Die Schulen der Psychotherapie befassen sich mit der individuellen Lebensgeschichte ohne die in der heutigen Zeit notwendige Berücksichtigung der Abhängigkeit des Individuums von den spezifischen Zeitqualitäten und vor allem den überindividuell bedingten Zeitperspektiven. Die individuelle «Lebensgeschichte» wird in den wenigsten Fällen explizit mit den Problemen der Zeit in Beziehung gebracht. Die psychotherapeutischen Konzepte sind aus einem gewissen Zeitgeist entstanden, haben es aber verpasst, sich an die zeitlichen Wandlungen anzupassen.

Ein Versuch, die menschliche Existenz und deren pathologische Manifestationen als einen in die $\mathrm{Zu}$ kunft weisenden Prozess aufzufassen, wurde durch Günther Ammon unternommen. Ammon bekundet einen gesellschaftlichen Optimismus, den man auch mit der Psychiatriereform der 70er Jahre in Verbindung bringen kann, der aber den fundamentalen Gegebenheiten des ausgehenden 20. Jahrhunderts nicht mehr entspricht. Nur aus der eigenen Erfahrung grossen Leides und Schmerzes kann eine solche Hoffnung für die Zukunft des Menschen entstehen. In seiner Funktion als Psychotherapeut stand ihm kein anderer Weg offen, als den Menschen aufzurufen, seine Energien zu sammeln für eine bessere Zukunft. Solche Heilsvorstellungen sind tiefverankert im Wesen der Psychotherapie, deren Wurzeln auf die Traditionen von Schamanen, Medizinmännern und Priestern zurückgehen.

In einem Überblick über die Dynamische Psychiatrie der Berliner Schule G. Ammons stellt I. Urspruch 
fest, dass psychisches Kranksein immer mit einer destruktiven oder defizitären Behinderung der Persönlichkeits- d. h. Identitätsentwicklung verbunden wäre. Diese Aussage muss heute im Lichte des beginnenden 21. Jahrhunderts interpretiert werden.

Die menschliche Gesellschaft befindet sich in einer andauernden prozesshaften Entwicklung, an der alle Individuen teilnehmen, und der Therapeut nicht weniger selbst in die geistig- moralische Evolution seiner Epoche eingebunden ist. Gesellschaft, Individuum und Psychiater bilden so ein Makrosystem, in dem jedes Element einen Einfluss auf das andere ausübt. Die Psychotherapieformen des vergangenen Jahrhunderts können nicht ohne weiteres in die Gegenwart übernommen werden, sondern bedürfen der Anpassung an die neuen gesellschaftlichen Gegebenheiten. Ein solcher Anspruch an die Therapie ist heute umso wichtiger, als sich die Gesellschaft in relativ kurzer Zeit mit exponentieller Geschwindigkeit verändert hat, und die individuellen Beziehungen in nichts mehr jenen vom Anfang des 20. Jahrhunderts gleichen.

Es scheint nun aber, dass solche Anpassungen der Psychotherapie an den neuen Menschen des 21. Jahrhunderts bisher nicht stattgefunden hätten. Für diese den individuellen ursprünglichen Bedürfnissen des Menschen. Es ist zu erwarten, dass der Trend zu einer kollektiven gesellschaftlichen Organisation mit einem autoritären Gebot der Selbstaufgabe des Individuums und nach dessen Einordnung in das gesellschaftliche Ganze noch lange weiter fortschreiten wird.

Wie lässt sich entscheiden, welche Form von Psychotherapie für einen Menschen adäquat wäre? Die Entscheidung über die zu verwendende therapeutische Methode sollte von mehreren Faktoren abhängig gemacht werden, wobei wir hier nur jene sozialer Art anführen. Neben der individuellen Pathologie des Patienten ist für die Therapiewahl seine nähere familiäre und berufliche Umgebung zu berücksichtigen. Da der Patient ebenso gut wie der Therapeut sich auch in einem Abhängigkeitsverhältnis vom gesamten gesellschaftlichen Kontext befindet, muss derselbe ebenfalls mitberücksichtigt werden. In einer Zeit der geschichtlichen Wende und der Frage nach einer Weiterexistenz des Menschen in einer bedrohten Umwelt ist die Beantwortung dieser letzten Frage besonders schwierig. Während in der Vergangenheit für eine Psychotherapie ein bipolares Verhältnis zwischen Patient und Therapeut bestand, sind die Ver-

\section{«Wie lässt sich entscheiden, welche Form von Psychotherapie für einen Menschen adäquat wäre?»}

Entwicklungshemmung der Psychotherapie sind mehrere Faktoren verantwortlich zu machen: die beinahe revolutionäre Veränderung der Gesellschaftsstrukturen und der Stellung des Einzelnen innerhalb derselben. Daneben können die Psychotherapien mit ihren Theorien Dogmen verglichen werden, die nur in der Folge grosser Erschütterungen eine Reform zulassen. Die Psychotherapeuten sind im Denken in ihren verschiedenen Schulen verankert und können sich nicht leicht von der Anziehung durch die Gruppe entziehen, aus ihrer Gruppenzugehörigkeit lösen. Im Gebiet der Geisteswissenschaften erfolgt der Fortschritt meistens wesentlich langsamer als in jenem der Naturwissenschaften, die rascher einen fassbaren und oft ökonomischen Vorteil $\mathrm{zu}$ verschaffen versprechen.

Die Menschheitsgeschichte ist seit wenigen Jahrzehnten in eine neue Epoche eingetreten, die auf die seelische Entität des Menschen einen enormen Einfluss ausübte durch die Infragestellung und Reduktion der individuellen Identität. Durch den Wandel des Umfeldes des Individuums sind seine charakterlichen Strukturelemente immer mehr von einem sukzessiven Abbau betroffen worden. Das Individuum löst sich immer mehr in der gesellschaftlichen Vereinigung auf, die neuen, vorwiegend materiellen Werten folgt, die mit der gesunden Entwicklung des Menschen nicht selten im Widerspruch stehen. Die kollektiven Werte haben einen Vorrang gegenüber hältnisse heute komplexer geworden. Der Therapeut und sein Klient sind heute mehr denn je eingefangen in einem ganzen umweltlichen Kontext und bilden zusammen ein dreipoliges, sich in Fortbewegung befindendes Kommunikationssystem.

Der Patient mit psychischen Problemen darf heute nicht nur im Hinblick auf seine individuellen Probleme, die oft äussere Ursachen haben, behandelt werden, sondern in seinem Bezug zur ganzen übrigen, in Bewegung befindlichen Umwelt, in der er sich befindet. Mit der individuellen Behandlung muss gleichzeitig auch auf die Umgebung eingewirkt werden. Die Idee von der reziproken Beeinflussung des Patienten und seines Milieus muss erweitert werden auf das gesamte kulturelle Milieu, was durch die allgemeine Anerkennung eines psychotherapeutischen Paradigmenwechsels möglich ist.

In einem Essay «Geschichte und Psychotherapie» bemerkt H. W. Schuch: «Wir begegnen in der Psychotherapie vielfältigen Zeitqualitäten. Probleme und Kunst der Psychotherapie bestehen nicht nur im Erkennen und lebensgeschichtlichen Verorten [...] individueller biographischer Mitteilungen, sondern auch in der spezifischen Handhabung [...] von therapeutischer Beziehung auf die spezifischen Zeitqualitäten.» Auf diese Forderung einzugehen, erscheint unter den gegebenen Milieubedingungen und deren unsicheren Perspektiven von essentieller Bedeutung.

Literatur auf Anfrage 